\title{
Heat Effects Occurring in the Supercooled Liquid State and Upon Crystallization of Metallic Glasses as a Result of Thermally Activated Evolution of Their Defect Systems
}

\author{
Andrei Makarov ${ }^{1,+} \mathbb{D}^{\mathbb{D}}$, Gennadii Afonin ${ }^{1, *,+} \mathbb{D}$, Yurii Mitrofanov ${ }^{1,+}$ and Nikolai Kobelev ${ }^{2,+}$ \\ and Vitaly Khonik $1,+$ \\ 1 Department of General Physics, State Pedagogical University, Lenin St. 86, Voronezh 394043, Russia; \\ a.s.makarov.vrn@gmail.com (A.M.); mitrofanovyup@gmail.com (Y.M.); v.a.khonik@vspu.ac.ru (V.K.) \\ 2 Institute of Solid State Physics, Russian Academy of Sciences, Chernogolovka, Moscow 142432, Russia; \\ kobelev@issp.ac.ru \\ * Correspondence: afoningv@gmail.com \\ + These authors contributed equally to this work.
}

Received: 20 February 2020; Accepted: 19 March 2020; Published: 24 March 2020

\begin{abstract}
We show that the kinetics of endothermal and exothermal effects occurring in the supercooled liquid state and upon crystallization of metallic glasses can be well reproduced using temperature dependences of their shear moduli. It is argued that the interrelation between the heat effects and shear modulus relaxation reflects thermally activated evolution of interstitial-type defect system inherited from the maternal melt.
\end{abstract}

Keywords: metallic glasses; heat effects; shear modulus; defects

\section{Introduction}

The non-crystallinity of glasses necessarily leads to the heat release or heat absorption upon heat treatment. This applies in full to metallic glasses (MGs) [1]. Exothermal effects in MGs take place upon structural relaxation below the glass transition temperature $T_{g}$ as well as upon crystallization. Strong endothermal reaction is observed in the supercooled liquid state, i.e., at temperatures $T_{g} \leq T<T_{x}$, where $T_{x}$ is the crystallization onset temperature [1]. Besides that, any physical impact (thermal cycling, plastic deformation, irradiation, etc.) leads to certain additional heat effects in MGs [2-4].

Current literature most often relates the heat effects in MGs to the changes of the free volume frozen-in upon glass production, as was originally suggested in the 1980s [5]. Later, the enthalpy relaxation in MGs was linked to the amount of the free volume in the simplest linear form [6] and subsequent experiments seemed to confirm this relationship [7,8]. As a matter of fact, heat effects in MGs are often considered as an indirect measure of the free volume since then [8].

However, it was repeatedly mentioned that the free volume concept has definite theoretical shortcomings [9] and the enthalpy release observed during relaxation cannot be simply interpreted as a measure of the free volume change [10]. On the other hand, exothermal reaction occurring upon crystallization is currently considered solely from a general viewpoint relating it to the appearance of crystalline phases, without discussing any specific details that might lead to the release of heat. We are unaware of any physical model, which could explain all heat effects taking place in the glassy state as well as upon crystallization within a unified physical concept, with the only exception discussed below.

Heat effects can be naturally explained if the defect system of glass is considered within the framework of the Interstitialcy theory (IT) suggested by Granato [11,12]. The IT argues that melting of 
metals is associated with the rapid increase of the concentration of interstitial defects in their most stable dumbbell form, in line with recent experimental observations [13,14]. These "defects" remain identifiable structural units in the liquid state [15] while rapid melt quenching freezes them in the solid glass. They retain all basic properties of dumbbell interstitials in crystals-high sensitivity to the applied shear stress, specific shear strain fields as well as characteristic low- and high-frequency vibration modes-although do not have any characteristic geometrical image like in crystals (two atoms trying to occupy the same lattice site) [16]. These entities can be considered as interstitial-type "defects" (quotation marks are omitted hereafter). Heat effects can be then interpreted in terms of the changes of the defect concentration. This approach provides quantitative explanations for quite a few other relaxation phenomena in MGs [17].

A description of the heat effects within the framework of the IT is based on an expression for the formation enthalpy of interstitial-type defects [11,12], $H=\alpha \Omega G$, where the dimensionless $\alpha \approx 1$ is related to the defect strain field [18], $\Omega$ is the volume per atom and $G$ is the unrelaxed shear modulus. The latter is related to the defect concentration $c$ as $G=\mu \exp (-\alpha \beta c)$, where $\mu$ is the shear modulus of the maternal crystal (i.e., the one, which was melted and then used for glass production by melt quenching) measured at the same temperature and the dimensionless shear susceptibility $\beta$ characterises the anharmonicity of the interatomic potential and by the definition equals the ratio of the fourth-order non-linear shear modulus to the second-order (i.e., "usual") shear modulus [17]. Typically, $\beta=15-20$ depending on MGs' chemical composition [17].

Thus, any change of the defect concentration leads to heat release/absorption depending on the $c$-change sign. For the glassy state, this mechanism was first suggested in Ref. [19]. It was later found that the whole excess enthalpy (heat content) of glass with respect to the maternal crystal (i.e., the difference between the heat contents of the glassy and crystalline states) within the IT framework is related to the elastic energy of interstitial-type defect system and this energy is fully released as heat when the defect concentration drops down to zero as a result of crystallization [20]. Thus, all exo- and endothermal heat effects occurring upon structural relaxation and crystallization can be considered as different sides of the same process-a change of interstitial-type defect concentration. In this case, the heat flow upon structural relaxation and crystallization of glass should be described by the same kinetic law, which relates the heat effects with the defect concentration. At that, the latter can be monitored by shear modulus measurements.

The two main equations of the IT given above lead to the difference in the heat flow of the glassy and crystalline states (per unit mass) conditioned by this relaxation mechanism [18]:

$$
\Delta W(T)=\frac{1}{m} \frac{d H_{g}(T)}{d t}=\frac{\dot{T}}{\beta \rho}\left[\frac{G(T)}{\mu(T)} \frac{d \mu(T)}{d T}-\frac{d G(T)}{d T}\right],
$$

where $H_{g}$ is the enthalpy of glass, $\dot{T}$ is the heating rate, $\rho$ is the density and $m$ is the sample's mass. It is seen that the heat flow $\Delta W$ is fully controlled by the shear moduli of glass and maternal crystal, $G(T)$ and $\mu(T)$. All other quantities are constants while any fitting parameters are absent. Equation (1) was successfully tested for the heat effects occurring in the glassy state [18] (A similar heat flow law was first derived in Ref. [19]. It differs from Equation (1) by slightly another expression in the square brackets: $\left[\frac{G_{r t}}{\mu_{r t}} \frac{d \mu(T)}{d T}-\frac{d G(T)}{d T}\right]$, where $G_{r t}$ and $\mu_{r t}$ are the shear moduli of glass and maternal crystal at room temperature, respectively. This heat flow law provides the results very close to those given by Equation (1). The latter, however, is more general [18]). However, as mentioned above, the same mechanism should be valid for the crystallization as well, as first argued in Ref. [18] and recently confirmed experimentally on a particular metallic glass [21]. In this work, we show that Equation (1) is valid both for the relaxation in the supercooled liquid state and the whole crystallization kinetics of four Zr-based MGs displaying diverse physical properties. 


\section{Experimental}

X-ray non-crystalline $\quad \mathrm{Zr}_{47.5} \mathrm{Cu}_{47.5} A l_{5}, \quad \mathrm{Zr}_{65} \mathrm{Cu}_{15} A l_{10} \mathrm{Ni}_{10}, \quad \mathrm{Zr}_{47} \mathrm{C} u_{45} A l_{7} \mathrm{Fe}_{1}$ and $\mathrm{Zr}_{52.5} \mathrm{Cu}_{17.9} \mathrm{Ni}_{14.6} \mathrm{Al}_{10} \mathrm{Ti} i_{5}$ (at.\%, labelled as $\mathrm{ZrAl}$, $\mathrm{ZrNi} i_{10}, \mathrm{ZrFe}_{1}$ and $\mathrm{ZrTi}_{5}$ hereafter) produced as $5 \times 2 \times(40-50) \mathrm{mm}^{3}$ bars were used for the investigation. The choice of these glasses was conditioned by the following reasons. First, all above Zr-based glasses (either in the initial or preannealed states) display a level of the mechanical damping in the supercooled liquid state (i.e., above $T_{g}$ ), which is small enough to ensure automatic measurements of the shear modulus (see below). As noted earlier, a high enough damping level above $T_{g}$ results in the loss of automatic resonant frequency tracking [21], which strongly limits the range of possible glass compositions. Second, these glasses have diverse and, in some sense, particular properties. Specifically, the $\mathrm{ZrNi} i_{10}$ glass is very resistant to oxidation [22] that strongly favours precise calorimetric measurements on relatively small samples and their subsequent comparison with calculation results. The glasses $\mathrm{ZrAl} 5$ and $\mathrm{ZrFe}_{1}$ display, respectively, big and small compression plasticity [23] that could potentially affect their high temperature behavior. The $\mathrm{ZrTi}_{5}$ glass is being produced industrially for different applications and demonstrates enhanced corrosion resistance [24]. Besides that, the choice of the above MGs is conditioned by the fact that they can be fully crystallized (without any additional phase transformations) below the maximal temperature $(\approx 900 \mathrm{~K})$ achievable upon standard calorimetric measurements. Thus, it is important to check the IT approach sketched above on MGs displaying diverse physical properties.

Heat effects were measured by a Hitachi DSC 7020 instrument in flowing $N_{2}(99.999 \%)$. The mass of the samples was 50-70 mg. Every DSC run on a glassy sample was taken up to the temperature of the full crystallization. It was next followed by the 2nd run on the same sample and the difference between the two runs was then calculated. It is this difference, which is shown below in Figure 2 and compared with $\Delta W$-calculations performed using Equation (1).

The electromagnetic acoustic transformation (EMAT) method (see Ref. [25] for the method's details) was applied to measure the transverse resonant frequencies $f(500-700 \mathrm{kHz})$ of $5 \times 5 \times 2 \mathrm{~mm}^{3}$ samples at temperatures of up to $810 \mathrm{~K}$ in a vacuum of $\approx 0.01 \mathrm{~Pa}$. For this purpose, frequency scanning was automatically performed every $10-15 \mathrm{~s}$ and the resonant frequency was determined as a maximal signal response received by the pick-up coil upon scanning. The half-width of the resonant curve was used to calculate the mechanical quality factor (equal to the inverse damping) and the latter was used to estimate the precision of resonant frequency determination. The shear modulus was then calculated as $G(T)=G_{r t} f^{2}(T) / f_{r t}^{2}$, where $f_{r t}$ and $G_{r t}$ are the vibration frequency and shear modulus at room temperature, respectively. This way of $G$-calculation ignores possible density changes that can occur upon heating (usually less than $1 \%$ ). The errors for the absolute $G_{r t}$-values were accepted to be $1-2 \%$. Then, the errors in the absolute $G(T)$ data are about the same while the error in the measurements of $G(T)$-changes was estimated to be $5 \mathrm{ppm}$ near room temperature and about $100 \mathrm{ppm}$ near $T_{g}$. A heating/cooling rate of $3 \mathrm{~K} / \mathrm{min}$ was accepted in all shear modulus and DSC measurements.

Three of the MGs under investigation $\left(\mathrm{ZrAl}_{5}, \mathrm{ZrNi}_{10}\right.$ and $\left.\mathrm{ZrFe}_{1}\right)$ were tested in the initial state. This was impossible for the fourth glass $\left(Z_{r T i_{5}}\right)$ because of the large damping near $T_{g}$, which results in the loss of EMAT automatic signal tracking. To avoid this effect, this MG was first annealed by heating into the supercooled liquid region and cooling back to room temperature. This MG is labelled as "relaxed" below. Both initial and relaxed MGs were first tested from room temperature up to temperatures of 780-810 K, which in all cases lead to the full crystallization. The second run for each glass was performed on the same sample in order to measure shear modulus $\mu(T)$ in the crystalline state.

\section{Results and Discussion}

Figure 1a-d shows temperature dependences of the shear modulus $G(T)$ for all MGs under investigation. The room-temperature shear moduli $G_{r t}$ listed in this Figure are taken from Ref. [26]. It is seen that $G(T)$-patterns for all MGs are quite similar. Heating of glassy samples leads to 
a monotonous decrease of $G$ while the slope $|d G / d T|$ decreases by several times near $T_{g}$, which constitutes a typical behavior upon high-frequency $G$-measurements [17]. Heating by $50-70 \mathrm{~K}$ above $T_{g}$ results in the beginning of crystallization, which leads to an increase of the shear modulus by $22 \%$ $\left(\mathrm{ZrFe}_{1}\right)$ to $40 \%\left(\mathrm{ZrNi}_{10}\right)$. In the crystalline state (2nd run), the shear modulus $\mu(T)$ demonstrates a featureless decrease with temperature.

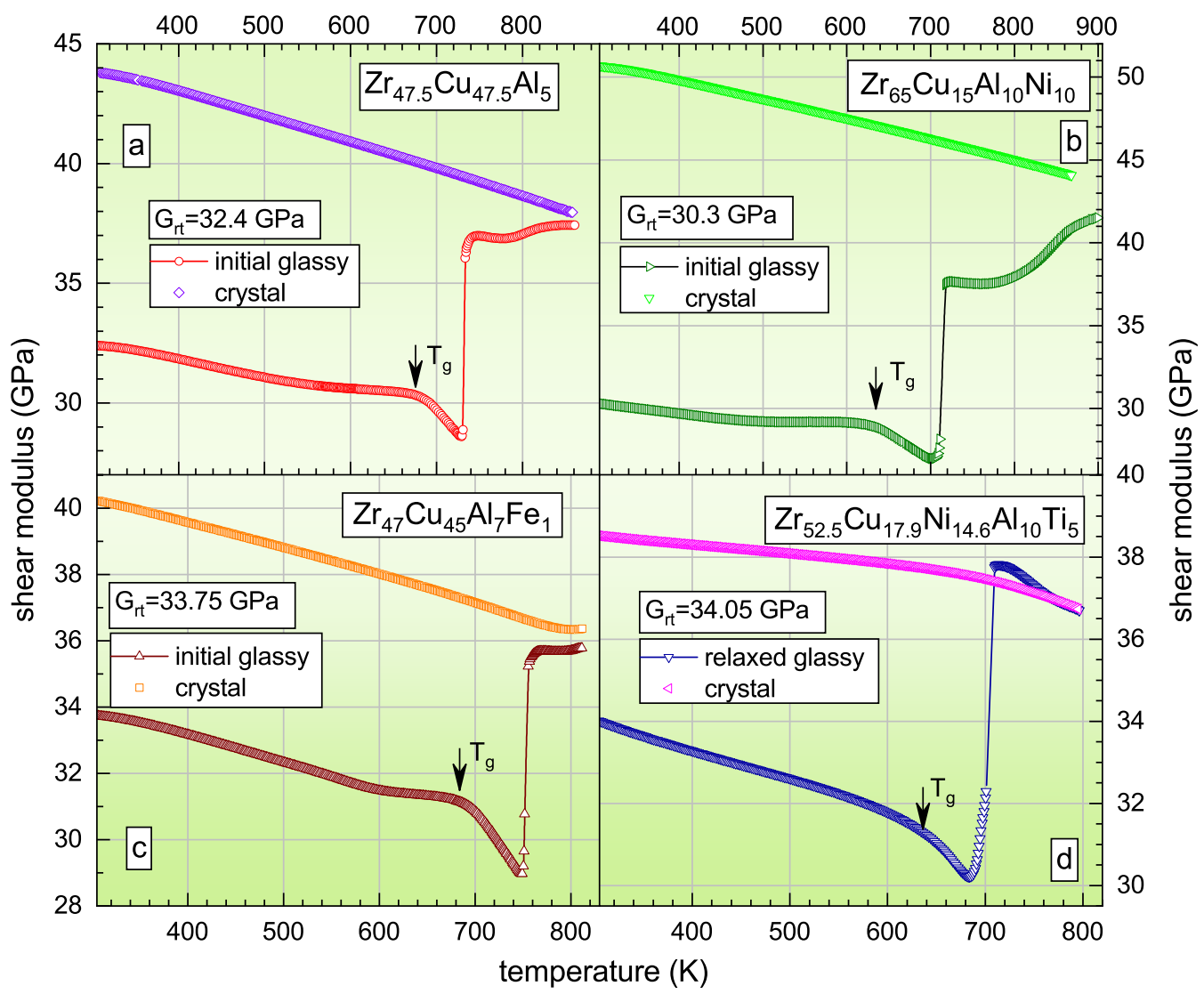

Figure 1. Temperature dependences of the shear modulus measured at $3 \mathrm{~K} / \mathrm{min}$ for the glasses under investigation in the initial state $(\mathbf{a}-\mathbf{c})$, after relaxation (d) and after full crystallization (a-d). The calorimetric $T_{g}$ 's are indicated by the arrows. The room-temperature shear moduli are taken from Ref. [26].

Figure 2 shows DSC traces of the MGs under investigation at the same heating rate of $3 \mathrm{~K} / \mathrm{min}$. Initial glasses $\left(\mathrm{ZrAl}_{5}, \mathrm{ZrNi}_{10}\right.$ and $\left.\mathrm{ZrFe}_{1}\right)$ demonstrate (i) exothermal reaction below $\mathrm{T}_{g}$ (not seen in the scale of Figure $2 \mathrm{a}-\mathrm{c}$ ), (ii) heat absorption in the supercooled liquid state (i.e., at temperatures $T_{g} \leq T<T_{x}$ ), which is bigger compared with that of the glassy and crystalline phases, and (iii) large heat release due to the crystallization. In the relaxed MG $\left(\mathrm{ZrTi}_{5}\right)$, the feature (i) is absent, as one would expect.

Figure 2 also gives the heat flow $\Delta W(T)$-curves calculated with Equation (1) at $\dot{T}=3 \mathrm{~K} / \mathrm{min}$ using the corresponding temperature dependences of the shear moduli $G(T)$ and $\mu(T)$ given in Figure 1. The way of the determination of the shear susceptibility $\beta$ from the experimental data and its values together with the densities for the MGS under investigation are given in Ref [26]. The insets in Figure 2 show experimental and calculated $\Delta W(T)$-curves in the supercooled liquid and crystallization temperature regions on enlarged scales. Figure 2 in general demonstrates that the calculation provides a rather good quantitative reproduction of the experimental $\Delta W(T)$-curves. First of all, this applies to the heat absorption for all glasses in the supercooled liquid state. Temperature position of the crystallization exothermal peak is reproduced within $\approx 10 \mathrm{~K}$ for $\mathrm{ZrNi} i_{10}$ and $\leq 6 \mathrm{~K}$ for other glasses. The height of this peak is reproduced within $10 \%-15 \%$ accuracy with the exception of 
$\mathrm{ZrFe}_{1}$ for which the calculation gives about $60 \%$ of the experimental height (not shown in Figure 2). It should be emphasized that the density of data points provided by our EMAT system in the range of fast crystallization (i.e., upon a rapid change of the shear modulus) is significantly smaller than that for temperatures $T<T_{x}$ (see Figure 1 ) and this constitutes a significant source for the calculation errors when using Equation (1), which contains both derivatives of the shear moduli and their difference. Nonetheless, one can conclude that this equation provides a good description of the heat effects on the basis of shear modulus data. It is interesting to note that the calculation reproduces the fine details of the crystallization kinetics as exemplified by the data around $T=700 \mathrm{~K}$ shown in the inset of Figure 2d.

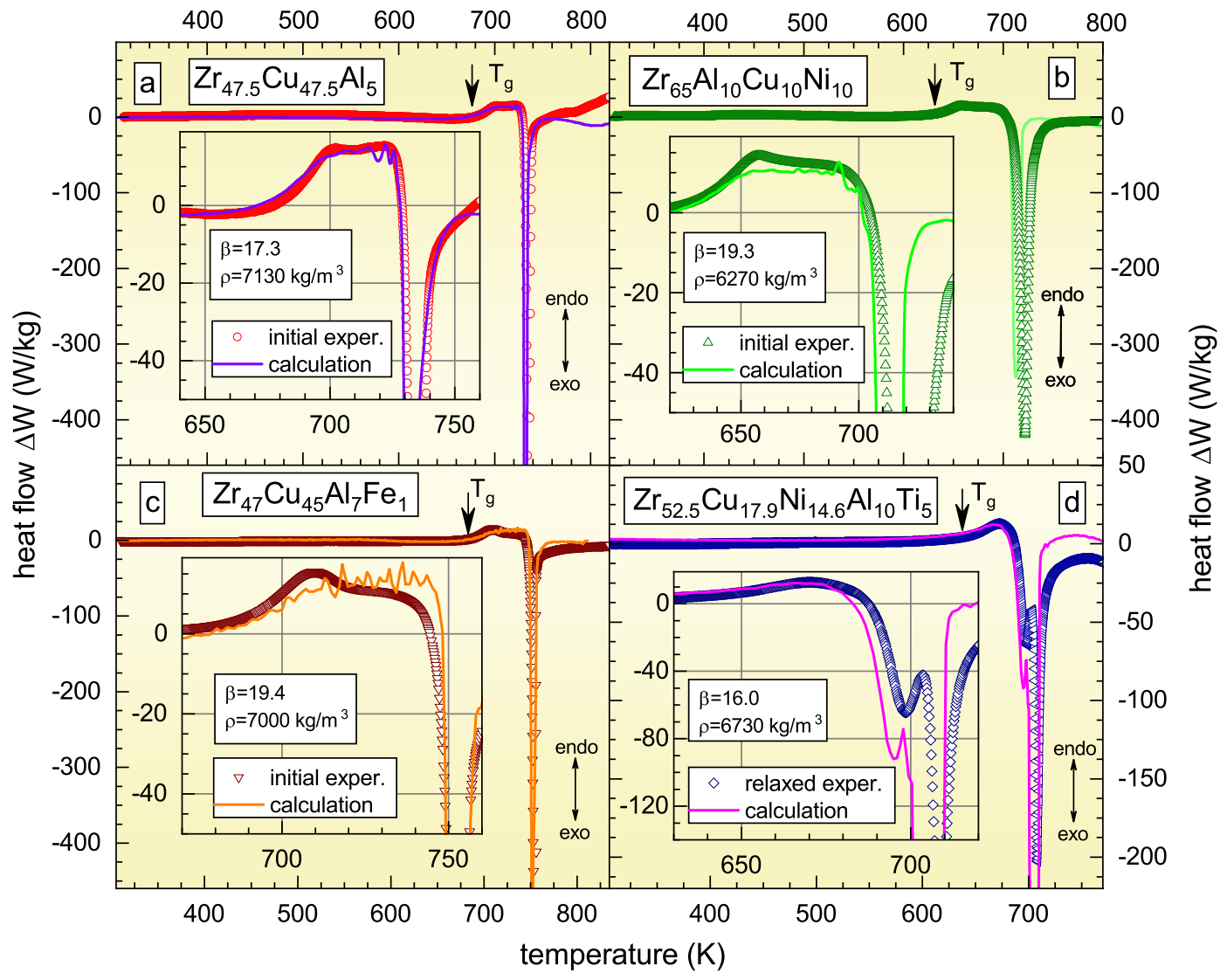

Figure 2. Experimental and calculated using Equation (1) DSC traces for the MGs under investigation in the initial $(\mathbf{a}-\mathbf{c})$ and relaxed states $(\mathbf{d})$. The insets show the heat flow in the supercooled liquid state and upon crystallization on an enlarged scale. The shear susceptibilities $\beta$ and the densities $\rho$ are taken from Ref. [26].

The obtained results confirm the basic idea of the IT sketched above: the origin of the heat absorption and heat release occurring in the glass transition range as well as upon crystallization can be understood as a result of the change of the concentration of interstitial-type defects frozen-in from the melt upon glass production. In particular, structural relaxation below $T_{g}$ provides relatively small decrease of the defect concentration and constitutes the reason of moderate exothermal heat effect (feature (i) mentioned above). The defect concentration above $T_{g}$ increases with temperature leading to the heat absorption (feature (ii)). Finally, fast crystallization at $T>T_{x}$ results in a rapid drop of the defect concentration down to zero providing quick dissipation of the defect elastic energy into heat, which is released as a strong crystallization peak (feature (iii)) [20].

As mentioned above, the interstitial defects in crystalline structures are unambiguously geometrically defined as two atoms trying to occupy the same lattice site. However, the situation in metallic non-crystalline structures is more complex. Molecular static simulation performed on a 
monoatomic glassy structure revealed localized nano-regions, which display the properties similar to those of dumbbell interstitials in simple metals [16]. These findings imply that interstitial-type defects can indeed exist in a monatomic non-crystalline structure. The issue whether such structural entities can be found in polyatomic glassy structures constitutes a major challenge for further research in this direction.

\section{Conclusions}

The investigation of four Zr-based glasses displaying diverse physical properties showed that the heat effects occurring in the supercooled liquid state and upon crystallization can well reproduced with Equation (1) using experimentally determined temperature dependences of shear moduli of the glass and maternal crystal. This equation is derived on the basis of the Interstitialcy theory and assumes that the heat effects in all temperature ranges take place due to the thermally activated change of the concentration of interstitial-type defects frozen-in from the melt upon glass production. A change of the defect concentration leads to a release or absorption of the total defect formation enthalpy constituting the physical reason for the heat effects both in the glassy state and upon crystallization.

Author Contributions: All authors equally contributed to this work. All authors have read and agreed to the published version of the manuscript.

Funding: This research was supported by the Russian Science Foundation under the grant 20-62-46003.

Conflicts of Interest: The authors declare no conflict of interest.

\section{References}

1. Chen, H.S. Glassy metals. Rep. Prog. Phys. 1980, 43, 353. [CrossRef]

2. Saida, J.; Yamada, R.; Wakeda, M.; Ogata, S. Thermal rejuvenation in metallic glasses. Sci. Technol. Adv. Mater. 2017, 18, 152-162. [CrossRef] [PubMed]

3. Ebner, C.; Escher, B.; Gammer, C.; Eckert, J.; Pauly, S.; Rentenberger, C. Structural and mechanical characterization of heterogeneities in a CuZr-based bulk metallic glass processed by high pressure torsion. J. Alloys Compd. 2018, 160, 147-157. [CrossRef]

4. Pan, J.; Wang, Y.X.; Guo, Q.; Zhang, D.; Greer, A.L.; Li, Y. Extreme rejuvenation and softening in a bulk metallic glass. Nat. Commun. 2018, 9, 560. [CrossRef] [PubMed]

5. van den Beukel, A.; Radelaar, S. On the kinetics of structural relaxation in metallic glasses. Acta Metall. 1983, 31, 419-427. [CrossRef]

6. van den Beukel, A.; Sietsma, J. The glass transition as a free volume related kinetic phenomenon. Acta Met. Mater. 1990, 38, 383-389. [CrossRef]

7. Slipenyuk, A.; Eckert, J. Correlation between enthalpy change and free volume reduction during structural relaxation of $\mathrm{Zr}_{55} \mathrm{Cu}_{30} \mathrm{Al}_{10} \mathrm{Ni}_{5}$ metallic glass. Scr. Mater. 2004, 50, 39-44. [CrossRef]

8. Haruyama, O.; Nakayama, Y.; Wada, R.; Tokunaga, H.; Okada, J.; Ishikawa, T.; Yokoyama, Y. Volume and enthalpy relaxation in $\mathrm{Zr}_{55} \mathrm{Cu}_{30} \mathrm{Ni}_{5} \mathrm{Al}_{10}$ bulk metallic glass. Acta Mater. 2010, 58, 1829-1836. [CrossRef]

9. Miracle, D.B.; Egami, T.; Flores, K.M.; Kelton, K.F. Structural Aspects of Metallic Glasses. MRS Bull. 2007, 32, 629-634. [CrossRef]

10. Cheng, Y.Q.; Ma, E. Indicators of internal structural states for metallic glasses: Local order, free volume, and configurational potential energy. Appl. Phys. Lett. 2008, 93, 051910. [CrossRef]

11. Granato, A.V. Interstitialcy model for condensed matter states of face-centered-cubic metals. Phys. Rev. Lett. 1992, 68, 974-977. [CrossRef] [PubMed]

12. Granato, A.V. Interstitialcy theory of simple condensed matter. Eur. J. Phys. 2014, 87, 18. [CrossRef]

13. Safonova, E.V.; Mitrofanov, Y.P.; Konchakov, R.A.; Vinogradov, A.Y.; Kobelev, N.P.; Khonik, V.A. Experimental evidence for thermal generation of interstitials in a metallic crystal near the melting temperature. J. Phys. Cond. Matter 2016, 28, 215401. [CrossRef] [PubMed]

14. Goncharova, E.V.; Makarov, A.S.; Konchakov, R.A.; Kobelev, N.P.; Khonik, V.A. Premelting generation of interstitial defects in polycrystalline indium. J. Exp. Theor. Phys. Lett. 2017, 106, 35-39. [CrossRef] 
15. Nordlund, K.; Ashkenazy, Y.; Averback, R.S.; Granato, A.V. Strings and interstitials in liquids, glasses and crystals. Europhys. Lett. 2005, 71, 625-631. [CrossRef]

16. Goncharova, E.V.; Konchakov, R.A.; Makarov, A.S.; Kobelev, N.P.; Khonik, V.A. Identification of interstitial-like defects in a computer model of glassy aluminum. J. Phys. Condens. Matter 2017, 29, 305701. [CrossRef]

17. Khonik, V.A.; Kobelev, N.P. Metallic glasses: A new approach to the understanding of the defect structure and physical properties. Metals 2019, 9, 605. [CrossRef]

18. Kobelev, N.P.; Khonik, V.A. Theoretical analysis of the interconnection between the shear elasticity and heat effects in metallic glasses. J. Non-Cryst. Sol. 2015, 427, 184-190. [CrossRef]

19. Mitrofanov, Y.P.; Makarov, A.S.; Khonik, V.A.; Granato, A.V.; Joncich, D.M.; Khonik, S.V. On the nature of enthalpy relaxation below and above the glass transition of metallic glasses. Appl. Phys. Lett. 2012, 101, 131903. [CrossRef]

20. Afonin, G.V.; Mitrofanov, Y.P.; Makarov, A.S.; Kobelev, N.P.; Wang, W.H.; Khonik, V.A. Universal relationship between crystallization-induced changes of the shear modulus and heat release in metallic glasses. Acta Mater. 2016, 115, 204-209. [CrossRef]

21. Mitrofanov, Y.P.; Wang, D.P.; Makarov, A.S.; Wang, W.H.; Khonik, V.A. Towards understanding of heat effects in metallic glasses on the basis of macroscopic shear elasticity. Sci. Rep. 2016, 6, 23026. [CrossRef] [PubMed]

22. Kim, C.W.; Jeong, H.G.; Lee, D.B. Oxidation of $Z_{65} A l_{10} N i_{10} C u_{15}$ bulk metallic glass. Mater. Lett. 2008, 62, 584-586. [CrossRef]

23. Wang, D.P.; Zhao, D.Q.; Ding, D.W.; Bai, H.Y.; Wang, W.H. Understanding the correlations between Poisson's ratio and plasticity based on microscopic flow units in metallic glasses. J. Appl. Phys. 2014, 115, 123507. [CrossRef]

24. Chieh, T.C.; Chu, J.; Liu, C.T.; Wu, J.K. Corrosion of $Z r_{52.5} C u_{17.9} N i_{14.6} A l_{10} T i_{5}$ bulk metallic glasses in aqueous solutions. Mater. Lett. 2003, 57, 3022-3025. [CrossRef]

25. Vasil'ev, A.N.; Gaidukov, Y.P. Electromagnetic excitation of sound in metals. Soviet Physics Uspekhi 1983 26, 952. [CrossRef]

26. Afonin, G.V.; Mitrofanov, Y.P.; Makarov, A.S.; Kobelev, N.P.; Khonik, V.A. On the origin of heat effects and shear modulus changes upon structural relaxation and crystallization of metallic glasses. J. Non-Cryst. Sol. 2017, 475, 48-52. [CrossRef]

(C) 2020 by the authors. Licensee MDPI, Basel, Switzerland. This article is an open access article distributed under the terms and conditions of the Creative Commons Attribution (CC BY) license (http:/ / creativecommons.org/licenses/by/4.0/). 